\title{
ENTEROPARASITOS EM MANIPULADORES DE MERENDA ESCOLAR EM CUITÉ, PARAÍBA
}

Francisco Patricio de Andrade Júnior

Mestrando em Produtos Naturais e Sintéticos Bioativos, Centro de Ciências da Saúde, Univesidade Federal da Paraíba, Brasil.

Thiago Willame Barbosa Alves

Farmacêutico, Universidade Federal de Campina Grande, Brasil.

\section{Edson Douglas Silva Pontes}

Graduando em Nutrição, Unidade Acadêmica de Saúde, Universidade Federal de Campina Grande, Brasil.

Vanessa Santos de Arruda Barbosa

Docente $\operatorname{Dr}^{\mathrm{a}}$. Unidade Acadêmica de Saúde, Universidade Federal de Campina Grande, Brasil.
RESUMO: O presente estudo teve como objetivo avaliar a prevalência de enteroparasitos e as variáveis epidemiológicas associadas à infecção parasitária em manipuladores de merenda escolar de Cuité-PB. As amostras fecais foram analisadas pelo método de centrífugo-sedimentação simples e houve aplicação de questionário semiestruturado em manipuladores de merenda escolar de 12 escolas do município de Cuité. Foi utilizado o teste qui-quadrado e as variáveis foram analisadas a partir do Software Statistical Package for Social Sciences.Dos 57 manipuladores de merenda escolar analisados, $78,9 \%$ estavam infectados por enteroparasitos/enterocomensais. Endolimax nana foi a espécie mais prevalente $(53,4 \%)$. Em relação à caracterização da amostra, $100 \%$ dos infectados eram do sexo feminino, $48,9 \%$ possuíam baixa escolaridade, $53,3 \%$ tinham renda familiar de até um salário mínimo e 60\% tinham entre 20-49 anos, ademais $40 \%$ dos infectados estavam bi/poliparasitados. Diante da alta prevalência são necessárias melhorias sanitárias, educacionais, no treinamento em boas práticas de manipulação, bem como a realização periódica do EPF.

PALAVRAS-CHAVE: Parasitas, Parasitologia, Epidemiologia.

\section{ENTEROPARASITES IN HANDLERS OF SCHOOL MEALS IN CUITE, BRAZIL}

ABSTRACT: Current study evaluates the prevalence of enteroparasites and epidemiological variables associated with parasite infections in handlers of school meals in Cuité PB Brazil. Fecal samples were analyzed by simple centrifugal-sedimentation method and a halfstructured questionnaire was applied to handlers of schools meals from two government-run schools in Cuité. The $\chi^{2}$ test was employed and variables were investigated by Software Statistical Package for Social Sciences. Further, $\mathbf{7 8 . 9 \%}$ of 57 meal handlers analyzed were infected by enteroparasites/enterocomensals. Endolimax nana was the prevalent species $(53.4 \%)$. All infected persons were female; 48.9\% had low schooling level; $53.3 \%$ earned up to one minimum wage and $60 \%$ were within the $20-49$ years age bracket. Forty percent were bi/polyparasited. In the wake of high prevalence, better sanitary, educational improvements are required, coupled to good handling practices and the periodical EPF tests.

KEY WORDS: Parasites, Parasitology, Epidemiology. 


\section{INTRODUÇão}

As doenças transmitidas por alimentos (DTAs) são importantes causas de morbidade e mortalidade em todo o mundo, tendo emergido, nas últimas duas décadas, como um problema econômico e de saúde pública ${ }^{1}$.

Estas enfermidades podem surgir a partir da contaminação de alimentos por agentes biológicos (bactérias, fungos, helmintos, protozoários e vírus), químicos (como ácidos, bases e solventes) e físicos (como pedras e pregos) ${ }^{2}$. Contudo, dentre os possíveis agentes contaminantes de alimentos do tipo biológico, há destaque para os parasitos intestinais, responsáveis por causar enteroparasitoses, que podem ser obtidas diretamente por rota fecal-oral ou indiretamente por meio de alimentos e água contaminados ${ }^{3}$.

Em países subdesenvolvidos e em desenvolvimento, como o Brasil, diversos fatores atuam como facilitadores para a transmissão de enteroparasitos, a exemplo do baixo saneamento básico, a falta de higiene corporal, a baixa escolaridade e a impossibilidade ao acesso a água de qualidade ${ }^{4}$. Na região Nordeste do Brasil, por sua vez, as enteroparasitoses são frequentes, sobretudo em crianças, pelas precárias condições socioeconômicas e educacionais onde estas estão inseridas, assim como, em razão da má higienização de alimentos ou até mesmo da incorreta manipulação pelos manipuladores de alimentos de merenda escolar que encontrem-se infectados ${ }^{4,5}$, sendo estes profissionais alvos de diversos estudos epidemiológicos pela comprovada possibilidade de serem ferramentas para o surgimento de DTAs ${ }^{4,6}$.

Dessa forma, para efeito da inspeção sanitária de alimentos, qualquer pessoa que entre direta ou indiretamente em contato com substâncias alimentícias é considerada manipulador. As merendeiras entram nesse grupo porque são as profissionais responsáveis por todo o processo de produção das refeições escolares ${ }^{7}$.

A contaminação das merendeiras por parasitos intestinais pode ocasionar no aumento da probabilidade de contaminação das merendas escolares seja pelo mautreinamento, inadequadas condições higienicossanitárias ou até mesmo o contato interpessoal entre outros funcionários e estudantes, podendo acometer aqueles que consomem estes alimentos ${ }^{8,9}$.
Nesse contexto, as merendeiras podem desempenhar importante papel na transmissão de doenças, uma vez que pelo contato direto e constante com os alimentos há maior facilidade de carrear agentes patogênicos a estes insumos ${ }^{6,10}$.

Assim, o presente estudo teve como objetivo avaliar a prevalência de enteroparasitos e as variáveis epidemiológicas associadas à infecção parasitária em manipuladores de merenda escolar de Cuité-PB.

\section{METODOLOGIA}

\section{TIPO DE ESTUDO}

Tratou-se de um estudo do tipo descritivo, analítico e transversal, em que foi realizado um inquérito coproparasitoscópico em amostras de manipuladores de alimentos de escolas públicas do município de Cuité no período de agosto a dezembro de 2017. Ademais, houve a aplicação de um questionário semiestruturado para se traçar o perfil socioeconômico e higienicossanitário da população estudada e se avaliar as variáveis epidemiológicas associadas aos infectados. O trabalho foi aprovado pelo Comitê de Ética em Pesquisa do Hospital Universitário Alcides Carneiro-UFCG (parecer $\left.n^{\mathrm{o}} 1.258 .124\right)$.

\section{Caracterização da amostra}

A amostragem foi do tipo não probabilística de conveniência ${ }^{11}$, no qual foram abordados manipuladores de merendas escolares, de ambos os sexos e todas as idades, em 12 creches e escolas municipais e estaduais da zona urbana do município de Cuité - PB. Dentre os profissionais responsáveis por manipular e/ou preparar alimentos que participaram da pesquisa têmse as cozinheiras, auxiliares de cozinheira, auxiliares de serviços gerais (ASGs), além de monitoras e auxiliares de monitoras, sendo estas últimas profissionais e suas auxiliares as responsáveis por lecionar, cuidar e alimentar as crianças, tendo portanto contato direto com o alimento, sobretudo, quando se tratava de frutos e bolachas. 


\section{Critérios de inclusão e exclusão da amostra}

Os critérios adotados para a inclusão dos manipuladores de alimentos na pesquisa foram: entendimento dos objetivos, riscos e benefícios da pesquisa, concordância em participar assinando o Termo de Consentimento Livre e Esclarecido (TCLE); responder ao questionário para coleta de informações epidemiológicas e se prontificar em colher as amostras de fezes. Os mesmos foram orientados quanto aos procedimentos padrões de coleta de fezes e que durante o período de coleta das amostras não poderiam utilizar antiparasitários (exceto sob indicação médica). Foram excluídos do grupo de pesquisa os indivíduos que não compreenderam os possíveis riscos e benefícios da pesquisa e que não assinaram o TCLE.

\section{Área de estudo}

O município de Cuité (6028'53,94" S e 36008'58,87” W) está localizado na mesorregião do agreste paraibano e na microrregião do Curimataú Ocidental. De acordo com o censo do Instituto Brasileiro de Geografia e Estatística (IBGE) em 2017 esta cidade possuía 20.348 habitantes. Sua área territorial é de 758 $\mathrm{km}^{2}$, densidade $26,3 \mathrm{hab} . \mathrm{km}^{2}$ e apresenta-se distante da capital do Estado, João Pessoa, a 235 km, e da capital Federal, Brasília, a $2.713 \mathrm{~km}$. Sua altitude em relação ao nível do mar é de $667 \mathrm{~m}$ e possui como municípios limítrofes Cacimba de Dentro, Barra de Santa Rosa, Nova Floresta, Picuí, Pedra Lavrada, Cubatí e Sossego ${ }^{12}$.

\section{Coleta e processamento de dados}

Houve a aplicação de um questionário que apresentava perguntas abertas (em que a resposta era dada a partir da transcrição da fala do entrevistado) e fechadas (em que o entrevistado deveria optar por uma resposta pré-definada e contida no questionário). O mesmo teve como objetivo traçar o perfil de cada entrevistado com relação às suas condições socioeconômicas, o local de trabalho e seus conhecimentos sobre parasitose e boas práticas de manipulação. Em seguida foi feita a entrega de coletor universal para a coleta de amostra única de fezes.
O material biológico foi recolhido nas escolas em dia e horário marcado, acondicionado e transportado em caixa de isopor para o laboratório de Parasitologia do Centro de Educação e Saúde (CES). As amostras fecais, por sua vez, passaram por processamento por meio do método de centrífugo-sedimentação simples ${ }^{13}$. Todas as amostras foram analisadas em triplicata, por dois pesquisadores, utilizando o microscópico óptico com objetivas de 10x e $40 x^{14}$.

\section{Análise estatística dos dados}

Para a construção do banco as variáveis foram categorizadas da seguinte forma: idade - até 19 anos; 20 - 29 anos; 30 - 39 anos; 40 - 49 anos, 50 - 59 anos e acima de 60. A escolaridade foi categorizada em não escolarizado; baixa escolaridade - ensino fundamental incompleto ou completo; média escolaridade - ensino médio incompleto ou completo; e alta escolaridade . ensino superior incompleto ou completo. O tipo de esgotamento sanitário em adequado - esgoto geral; e inadequado - para fossa ou enterra. O abastecimento de água no domicílio e no local de trabalho: de procedência - rede pública; e sem procedência - poço ou comprada de caminhão pipa. A água utilizada para beber e no preparo dos alimentos foi categorizada em adequada - água mineral, filtrada ou fervida; e inadequada - água coada, clorada, sem tratamento ou de rede pública sem tratamento doméstico. Em relação ao destino do lixo considerou-se correto - coletado ou queimado; e incorreto - enterrado, usado como adubo ou jogado em terreno baldio. Além disso, ainda foi questionado aos manipuladores se os mesmos criavam animais e, se sim, quais seriam.

Para aqueles manipuladores que foram acometidos por alguma parasitose, foi considerado correto, quando a doença citada era realmente uma parasitose, ou seja, alguma helmitose ou protozoose e incorreto quando se tratava de outras doenças que não eram consideradas parasitoses intestinais. O tempo de realização de algum exame parasitológico de fezes foi categorizado em menor ou igual a seis meses e acima de seis meses, de acordo com a lei $\mathrm{n}^{0} 7.587^{15}$. Na questão aberta sobre como se pega uma parasitose, a classificação 
foi considerada correta quando o entrevistado afirmava que era possível adquirir parasitoses por meio da ingesta de água e/ou alimentos contaminados, contato com fezes e andar descalço e incorreta quando não citava nenhum dos fatores descritores anteriormente. Enquanto que para aqueles que se alimentavam de verduras cruas, o modo de lavá-las antes de consumir foi classificado em correto quando se respondia na pergunta aberta, que se fazia a lavagem mecânica em água corrente e deixava-se a verdura em repouso no hipoclorito de sódio, e incorreto, quando não se realizava um ou ambos os processos.

Para avaliar o nível de conhecimento sobre as boas práticas de manipulação, as respostas das questões sobre como pode ocorrer a contaminação dos alimentos, como as mãos podem contaminar o alimento, quais as doenças que os alimentos podem causar e o que considera importante na higiene pessoal, foram categorizadas em correta e incorreta. E quando perguntado sobre o último treinamento que participou, as categorias foram de menos de um ano e mais de um ano.

Os dados dos resultados coproparasitológicos e dos questionários foram computados em um banco de dados, no programa Statistical Package for Social Sciences (SPSS) versão 13.0, no qual se realizou a análise estatística. Na estatística descritiva, os dados das variáveis de maior relevância foram apresentados sob a forma de número absoluto e a frequência relativa calculada para a construção de tabelas.

Foram avaliadas associações estatísticas pelo teste Qui-quadrado entre as variáveis de risco e a presença de infecção parasitária, sendo aceito $\mathrm{p}<0,05$, estatisticamente significante, como critério para rejeição das hipóteses de nulidade.

\section{Encaminhamento para terapêutica medicamentosa e orientações}

Os resultados dos exames coproparasitológicos foram entregues aos manipuladores de merenda escolar, contendo as espécies parasitárias encontradas e a devidamente assinada e liberada por profissional qualificado (bióloga).

Os parasitados foram orientados a procurar profissional habilitado no programa Estratégia Saúde da
Família para tratamento antiparasitário.

\section{RESULTADOS}

Foram abordados 69 manipuladores, porém somente 57 aceitaram participar da pesquisa. Assim, dos 57 manipuladores de merenda escolar, 98,2\% eram do sexo feminino. A faixa etária predominante foi de 50 a 59 anos (36,8\%), seguido de 40 a 49 anos (33,3\%), 30 a 39 anos (24,6\%), 20 a 29 anos $(3,5 \%)$ e acima de 60 anos (1,8\%). Em relação ao estado civil, 45,6\% dos manipuladores de alimentos eram casados, seguido por solteiros $(31,6 \%)$, divorciados $(12,3 \%)$, união estável $(8,8 \%)$ e viúvos $(1,8 \%)$.

A renda familiar predominante entre a população estudada foi de até um salário mínimo com 57,9\%, seguido de um até dois salários mínimos com 36,8\% e acima de três salários mínimos com 5,3\%, enquanto que o nível de escolaridade foi predominantemente baixo (43,9\%), seguido de média escolaridade $(38,6 \%)$ e alta escolaridade (17,5\%).

Em relação às condições sanitárias no domicílio e local de trabalho dos manipuladores $80,7 \%$ apresentavam esgotamento inadequado, enquanto que em relação ao abastecimento de água no domićlío e água utilizada no local de trabalho $96,5 \%$ e $91,2 \%$, respectivamente, eram sem procedência. A respeito do destino do lixo, 3,5\% foi considerado inadequado, já a água para beber e a água usada no preparo dos alimentos foram consideradas inadequadas para 43,9\% e 70,2\% dos manipuladores, respectivamente.

Cerca de 57,9\% dos manipuladores afirmaram que criavam animais, sendo o cachorro o principal deles $(24,6 \%)$, seguido de cachorro e gato $(10,5 \%)$, gato $(5,3 \%)$, cachorro e galinha $(3,5 \%)$ e cachorro, gato e galinha $(3,5 \%)$, enquanto que outras combinações foram responsáveis por $10,5 \%$ dos entrevistados.

Em relação ao trabalho, dos 57 manipuladores, $56,1 \%$ afirmaram que este foi o primeiro emprego na cozinha, 38,6\% relataram ter tido outras experiências como cozinheiro(a), enquanto que 5,3\% optaram por não responder. Quanto ao consumo de alimentos crus, 15,8\% da população estudada afirmou consumir carne crua ou mal cozida, em relação às verduras, 91,2\% as consomem cruas. 
No exame parasitológico de fezes, dentre as 57 amostras fecais analisadas 78,9\% $(n=45)$ foram positivas. Destas 84,4\% estavam infectadas por enterocomensais e 15,6\% por enterocomensais e enteroparasitos. Na Figura 1 é possível observar as principais espécies de enteroparasitos/enterocomensais encontradas.

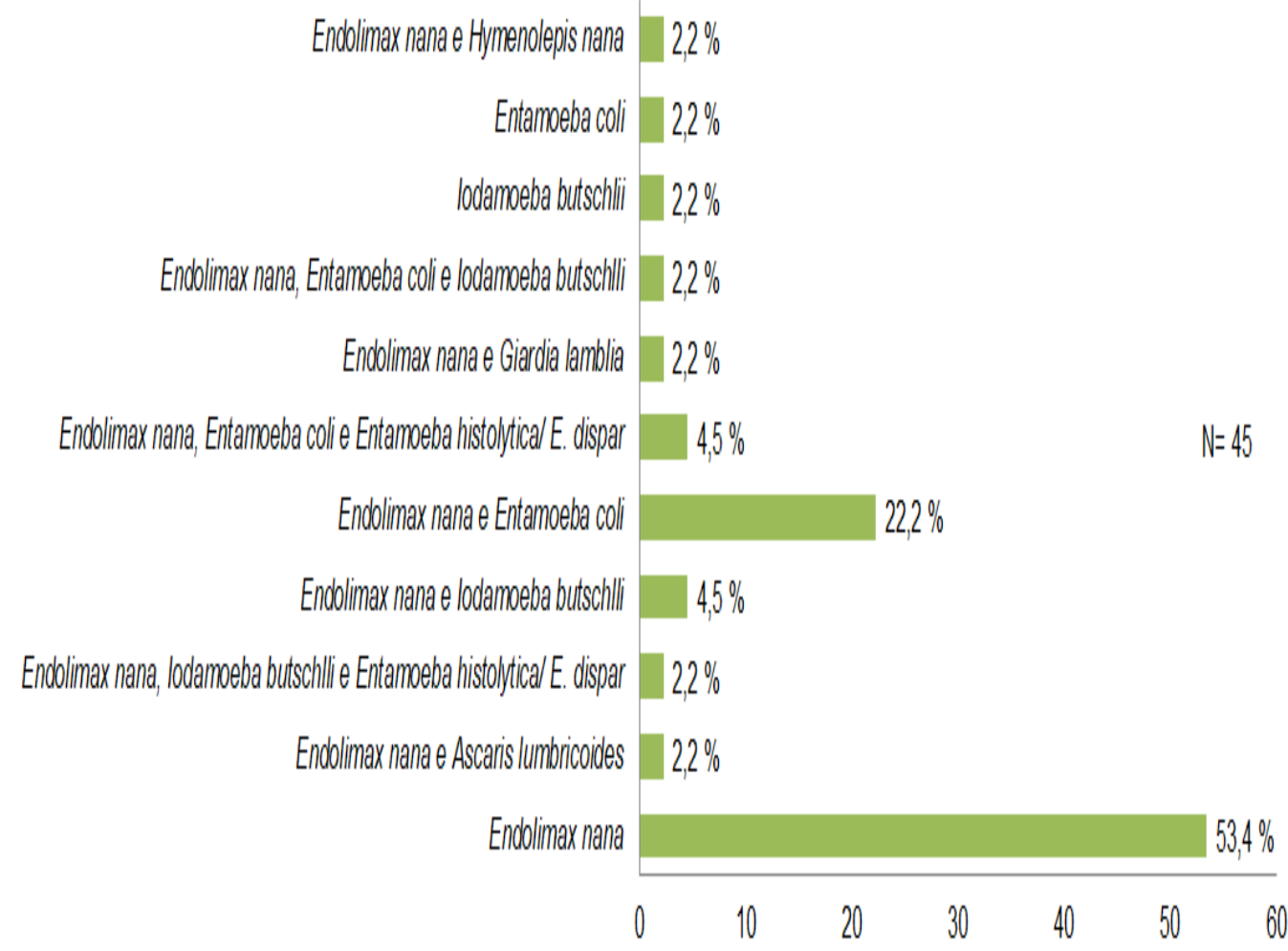

Figura 1. Prevalência das espécies de parasitos e/ou enterocomensais encontrados em manipuladores de alimentos de escolas públicas de Cuité-PB, 2017.

O monoparasitismo foi observado em $60 \%$ das amostras positivas, o biparasitismo em $31,1 \%$ e o poliparasitismo em $8,9 \%$.

Na Tabela 1 é mostrada a associação estatística entre dados socioeconômicos, higiene pessoal, conhecimentos sobre parasitoses e condições sanitárias do local de trabalho e domicílio com o resultado do exame parasitológico de fezes de manipuladores de merenda escolar de Cuité - PB, 2017.

Dos 57 manipuladores, 33,3\% afirmam ter tido alguma parasitose, porém somente 26,3\% destes procuraram o profissional médico e deram início à terapia farmacológica antiparasitária.

Na Tabela 2 observam-se a frequência dos manipuladores que já fizeram algum exame parasitológico e que já participaram de algum treinamento no trabalho. 
Tabela 1. Associação estatística entre dados socioeconômicos, higiene pessoal, conhecimentos sobre parasitoses e condições sanitárias do local de trabalho e domicílio com o resultado do exame parasitológico de fezes de manipuladores de merenda escolar de Cuité - PB, 2017

\begin{tabular}{|c|c|c|c|c|}
\hline \multirow[t]{2}{*}{ Categoria } & Positivo & \multicolumn{3}{|c|}{ Negativo } \\
\hline & $\mathbf{N}$ & $\%$ & $\mathbf{N}$ & $\%$ \\
\hline \multicolumn{5}{|l|}{ Dados socioeconômicos } \\
\hline \multicolumn{5}{|l|}{ Sexo* } \\
\hline Feminino & 45 & 100 & 11 & 91,7 \\
\hline Masculino & 0 & 0 & 1 & 8,3 \\
\hline Total & 45 & 100 & 12 & 100 \\
\hline \multicolumn{5}{|l|}{ Faixa etária\# } \\
\hline $20-49$ anos & 27 & 60,0 & 7 & 58,3 \\
\hline A partir de 50 anos & 18 & 40,0 & 7 & 41,7 \\
\hline Total & 45 & 100 & 12 & 100 \\
\hline \multicolumn{5}{|l|}{ Remuneração* } \\
\hline Até 1 salário mínimo & 24 & 53,3 & 9 & 75,0 \\
\hline$>$ de 1 salário mínimo & 21 & 46,7 & 3 & 25,0 \\
\hline Total & 45 & 100 & 12 & 100 \\
\hline \multicolumn{5}{|l|}{ Escolaridade* } \\
\hline Baixa escolaridade & 22 & 48,9 & 3 & 25,0 \\
\hline Média escolaridade & 16 & 35,6 & 6 & 50,0 \\
\hline Alta escolaridade & 07 & 15,6 & 3 & 25,0 \\
\hline Total & 45 & 100 & 12 & 100 \\
\hline \multicolumn{5}{|l|}{ Higiene pessoal } \\
\hline \multicolumn{5}{|c|}{ Lava as mãos após utilizar o banheiro* } \\
\hline Sim & 44 & 97,8 & 12 & 100 \\
\hline Não & 1 & 2,2 & 0 & 0 \\
\hline Total & 45 & 100 & 12 & 100 \\
\hline \multicolumn{5}{|c|}{ Forma de lavar as verduras cruas antes de consumi-las* } \\
\hline Correta $\uparrow$ & 23 & 51,1 & 7 & 58,3 \\
\hline Incorreta $\dagger$ & 18 & 40,0 & 4 & 33,3 \\
\hline Não consome & 4 & 8,9 & 1 & 8,4 \\
\hline Total & 45 & 100 & 12 & 100 \\
\hline \multicolumn{5}{|c|}{ Hábito de andar descalço* } \\
\hline Sim & 5 & 11,1 & 3 & 25,0 \\
\hline Não & 40 & 88,9 & 9 & 75,00 \\
\hline Total & 45 & 100 & 12 & 100 \\
\hline \multicolumn{5}{|c|}{ Fatores que considera importante na higiene pessoal* } \\
\hline Correto $\ddagger$ & 45 & 100 & 11 & 91,7 \\
\hline Incorreto $\$$ & 0 & 0 & 01 & 8,3 \\
\hline Total & 45 & 100 & 12 & 100 \\
\hline \multicolumn{5}{|c|}{ Conhecimentos sobre parasitoses } \\
\hline \multicolumn{5}{|c|}{ Sabe o que é parasitose?* } \\
\hline Sim & 27 & 60,0 & 9 & 75,0 \\
\hline Não & 18 & 40,0 & 3 & 25,0 \\
\hline Total & 45 & 100 & 12 & 100 \\
\hline \multicolumn{5}{|c|}{ Sabe como se pega parasitose?* } \\
\hline Sim & 23 & 51,1 & 8 & 66,7 \\
\hline Não & 22 & 48,9 & 4 & 33,3 \\
\hline Total & 45 & 100 & 12 & 100 \\
\hline
\end{tabular}




\begin{tabular}{|c|c|c|c|c|}
\hline & & & & (Conclusão) \\
\hline \multirow[t]{2}{*}{ Categoria } & \multicolumn{2}{|l|}{ Positivo } & \multicolumn{2}{|l|}{ Negativo } \\
\hline & $\mathbf{N}$ & $\%$ & $\mathbf{N}$ & $\%$ \\
\hline \multicolumn{5}{|c|}{ Ouviu falar em contaminação de alimentos* } \\
\hline Sim & 40 & 88,9 & 12 & 100 \\
\hline Não & 5 & 11,1 & 0 & 0 \\
\hline Total & 45 & 100 & 12 & 100 \\
\hline \multicolumn{5}{|c|}{$\begin{array}{l}\text { Com relação às mãos, elas podem contaminar os } \\
\text { alimentos?* }\end{array}$} \\
\hline Sim & 45 & 100 & 12 & 100 \\
\hline Não & 0 & 0 & 0 & 0 \\
\hline Total & 45 & 100 & 12 & 100 \\
\hline \multicolumn{5}{|c|}{ Como as mãos podem contaminar os alimentos?* } \\
\hline Correto $\S$ & 39 & 86,7 & 11 & 87,7 \\
\hline Incorreto $\S$ & 6 & 13,3 & 1 & 12,3 \\
\hline Total & 45 & 100 & 12 & 100 \\
\hline \multicolumn{5}{|c|}{$\begin{array}{l}\text { Você conhece a Lei de Qualidade Alimentar do Estado da } \\
\text { Paraíba?* }\end{array}$} \\
\hline Sim & 4 & 8,9 & 1 & 8,8 \\
\hline Não & 41 & 91,1 & 11 & 91,2 \\
\hline Total & 45 & 100 & 12 & 100 \\
\hline
\end{tabular}

Condições sanitárias do local de trabalho e domicílio

\begin{tabular}{|c|c|c|c|c|}
\hline \multicolumn{5}{|c|}{ Tipo de esgotamento sanitário* } \\
\hline Adequado & 9 & 20,0 & 2 & 16,7 \\
\hline Inadequado & 36 & 80,0 & 10 & 83,3 \\
\hline Total & 45 & 100 & 12 & 100 \\
\hline \multicolumn{5}{|c|}{ Abastecimento de água no domicílio* } \\
\hline Com procedência & 1 & 2,2 & 1 & 8,3 \\
\hline Sem procedência & 44 & 97,8 & 11 & 91,7 \\
\hline Total & 45 & 100 & 12 & 100 \\
\hline \multicolumn{5}{|c|}{ Abastecimento de água utilizada no trabalho* } \\
\hline Com procedência & 3 & 6,7 & 2 & 16,7 \\
\hline Sem procedência & 42 & 93,3 & 10 & 83,3 \\
\hline Total & 45 & 100 & 12 & 100 \\
\hline \multicolumn{5}{|c|}{ Destino do lixo do domicílio* } \\
\hline Adequado & 43 & 95,6 & 12 & 100 \\
\hline Inadequado & 2 & 4,4 & 0 & 0 \\
\hline Total & 45 & 100 & 12 & 100 \\
\hline \multicolumn{5}{|c|}{ Água utilizada para beber* } \\
\hline Adequado & 29 & 64,4 & 3 & 25,0 \\
\hline Inadequado & 16 & 35,6 & 9 & 75,0 \\
\hline Total & 45 & 100 & 12 & 100 \\
\hline \multicolumn{5}{|c|}{ Água utilizada no preparado de alimentos* } \\
\hline Adequado & 14 & 31,1 & 3 & 25,0 \\
\hline Inadequado & 31 & 68,9 & 9 & 75,0 \\
\hline Total & 45 & 100 & 12 & 100 \\
\hline
\end{tabular}

*Inaplicabilidade do teste Qui-Quadrado. $\uparrow$ correto quando se fazia a lavagem mecânica em água corrente e deixava-se a verdura em repouso com hipoclorito de sódio e incorreto, quando não se realizava um ou ambos os processos. mãos, higienização do corpo, cortar as unhas e outras medidas de higiene pessoal e incorreto quando nenhuma medida plausível foi citada. $\S$ correto quando se citava que as mãos não higienizadas em contato com os alimentos podia contaminá-los, incorreto quando se utilizavam outras justificativas. $\# \mathrm{p}=0,917$.

Fonte: Dados da pesquisa, 2017. 
Tabela 2. Realização de exame parasitológico e treinamento no trabalho em manipuladores de merenda escolar de Cuité - PB, 2017

\begin{tabular}{lcc}
\hline Categoria & N & $\%$ \\
\hline Já fez algum exame parasitológico de fezes? & & 3,5 \\
Não & 2 & 96,5 \\
Sim & 55 & 100 \\
\hline Total & 57 & 12,3 \\
\hline Há quanto tempo fez o EPF? & & 84,2 \\
Menor ou igual há 6 meses & 7 & 3,5 \\
Acima de 6 meses & 48 & 100 \\
Nunca fez & 2 & 57 \\
\hline Total & & 19,3 \\
\hline Quando foi o último treinamento que participou? & 11 \\
Há menos de um ano & 21 & 36,8 \\
Há mais de um ano & 25 & 43,9 \\
Nunca participou & 57 & 100 \\
\hline Total & & \\
\hline
\end{tabular}

Fonte: Dados da pesquisa, 2017.

\section{DISCUSSÃO}

Em Cuité-PB foi observada alta prevalência de positividade, na qual 78,9\% dos 57 manipuladores encontravam-se infectados por enteroparasitos/ enterocomensais. Essa foi a mais alta prevalência observada comparando-se a outras cidades brasileiras. Prevalências menores foram observadas no município de Campo Mourão-PR (50\% de 24 manipuladores de alimentos ${ }^{16}$; nos municípios mineiros de Luz e Dores Indaiá (27,3\% dos 22 manipuladores) ${ }^{17}$ e em Ângulo-PR (30\% dos 27 manipuladores) ${ }^{18}$.

Além disso, estudos realizados em outros países também têm demonstrado prevalências menores, como em um hospital terciário da Índia, em que de 84 manipuladores, $40,5 \%$ destes encontravam-se infectados, enquanto que de 210 manipuladores de restaurantes e fastfood do oeste iraniano $9 \%$ destes apresentavam-se infectados ${ }^{25,32}$.

Endolimaxnana foi a espécie mais prevalente nos manipuladores de merenda escolar de Cuité$\mathrm{PB}$, estando presente em 53,4\% das amostras fecais positivas. Resultados semelhantes foram observados nos municípios de Caxias do Sul-RS e Ribeirão PretoSP, em que $E$. nanaestava presente em $44,1 \%(\mathrm{n}=15) \mathrm{e}$ $54,9 \%(n=56)$ das amostras positivas de manipuladores, respectivamente ${ }^{19,20}$. Tais resultados divergiram dos encontrados em Campo Mourão-PR e Paraíba-PI em que Giardialambliae E. colifoi as espécies mais prevalentes respectivamente, em $21 \%(n=8)$ e $40,3 \%(n=52)$ de amostras fecais de manipuladores, respectivamente ${ }^{16,22}$. Pesquisas feitas em outros países também divergem dos resultados obtidos, em que se observou que em um hospital indiano, de 34 infectados 41,2\% apresentavamse com Entamoeba histolyta/E. dispar, enquanto que em manipuladores de fastfood e restaurantes do oeste iraniano, evidenciou-se que de 19 infectados, 47,4\% apresentaram E. coli em suas amostras fecais ${ }^{25,32}$.

A alta prevalência $E$. nana ,nas amostras fecais dos manipuladores, pode indicar deficientes medidas higiênicas ou ainda precariedade na qualidade da água e de saneamento básico ${ }^{21,23}$.

Dentre as associações parasitárias observadas, pode-se evidenciar a presença de agentes patogênicos como E.histolytica/E.dispar, G. lamblia e H. nana que podem ser facilmente difundidos aos alimentos e água ou rota fecal-oral e causar a contaminação dos consumidores. Também foi encontrado A. lumbricoides, em que a transmissão ocorre pela ingesta de ovos, geralmente, fixados em hortaliças que tenham sido cultivadas em locais próximos a fezes humanas ou tenham sido adubadas com esses excrementos.

Dos 45 manipuladores infectados no município de Cuité-PB, 100\% eram do sexo feminino. Resultados 
semelhantes foram observados no município de Campo Mourão, onde 12 manipuladores de merenda escolar infectados, $100 \%$ eram do sexo feminino ${ }^{16}$. Divergindo desses achados, menor percentual foi observado em Ribeirão Preto-SP, onde 142 manipuladores presentes em feiras livres, ruas e outros estabelecimentos vinculados ao município, $57 \%$ dos infectados eram do sexo feminino ${ }^{19}$ e em João Pessoa-PB, de 35 manipuladores positivos vinculados a um restaurante hospitalar, 64\% tratavam-se de mulheres ${ }^{24}$.

Em relação à faixa etária, Cuité-PB teve o predomínio de manipuladores infectados de 20 a 49 anos de idade (60\%). Assemelhando-se a esses resultados, no município de Parnaíba-PR, de 129 manipuladores de alimentos infectados, que se encontravam vinculados a restaurantes, $87 \%$ tinham entre 20 a 49 anos de idade ${ }^{6}$.

A respeito da faixa salarial dos manipuladores de alimentos em Cuité-PB que estavam positivos para enteroparasitos, observou-se que 53,3\% destes tinham como renda familiar, até um salário mínimo. Tal informação diverge dos dados encontrados em MaringáPR, onde 42 manipuladores infectados, 57,1\% possuíam de um a três salários mínimos como renda familiar ${ }^{18}$.

Quanto à escolaridade, o nível predominante entre os manipuladores de merenda escolar de Cuité-PB infectados foi de baixa escolaridade, como constatada em $48,9 \%$ dos participantes. Estes dados corroboram com um estudo realizado em Cascavel-PR, onde 131 manipuladores autônomos infectados, maior parte destes (65,6\%) possuíam baixa escolaridade ${ }^{20}$.

Pode-se inferir que o baixo nível de escolaridade e renda, predominantemente observadas em manipuladores de Cuité-PB, pode influenciar diretamente no conhecimento desses profissionais sobre parasitoses e suas medidas profiláticas. Isto pode ser evidenciado nesta pesquisa devido parte significativa dos profissionais positivos para enteroparasitos/enterocomensais não terem conhecimento sobre a correta lavagem de hortaliças (40\%) ou não saberem o que é parasitose (40\%) ou como adquiri-las (48,9\%).

Quanto à água do domicílio, 96,5\% dos manipuladores de merenda escolar de Cuité-PB afirmaram ser sem procedência, tal informação difere dos resultados encontrados em um estudo realizado em João Pessoa-PB, no qual de 67 manipuladores $91 \%$ confirmaram usufruir de água tratada, em seus domicílios ${ }^{24}$.

Aágua sem qualidade pode causar a contaminação por parasitos intestinais em mãos, alimentos, utensílios e equipamentos, tendo, portanto, ligação direta com a cadeia de transmissão. Ademais muitos enteroparasitos são transmitidos pela água e podem apresentar-se resistentes ao processo de cloração, fazendo com que populações utilizem água inadequada tornem-se mais vulneráveis à contaminação. Esta situação é preocupante em manipuladores do município de Cuité-PB, uma vez que $43,9 \%$ fazem o uso de águas que são consideradas inadequadas para consumo ${ }^{26}$.

Sobre o tipo de esgotamento sanitário, em CuitéPB, 80,7\% dos manipuladores apresentam esgotamento do tipo inadequado, o que se trata de um achado bastante negativo, uma vez que o destino incorreto dos dejetos contribui diretamente para o aumento da contaminação de seres humanos, por enteroparasitos ${ }^{27}$. Estes resultados divergem dos encontrados em João Pessoa-PB, em que de 67 manipuladores $56,7 \%$ apresentaram esgotamento sanitário adequado pelo uso do esgoto público ${ }^{24}$. Em relação ao destino do lixo, em Cuité-PB, 96,5\% dos manipuladores afirmaram que os seus detritos eram coletados ou queimados, sendo assim, considerados adequados; resultados semelhantes foram evidenciados em Parnaíba-PI onde 251 manipuladores, 94,8\% tinham o destino correto do lixo ${ }^{6}$. Tal informação torna-se interessante, uma vez que resíduos são importantes fatores de contaminação por agentes biológicos e de atração de vetores mecânicos, que contribuem para a disseminação massiva de enteroparasitos aos alimentos.

Outro importante fator facilitador para contrair enteroparasitoses é a criação de animais, com manejo inadequado, propiciando antropozoonoses, como a giardíase $^{28}$. Em Cuité-PB, 57,9\% dos manipuladores de merenda escolar afirmaram possuir animais de estimação, sendo o cachorro o principal deles (24,6\%).

Em relação ao consumo de alimentos crus, em Cuité-PB, 15,8\% dos manipuladores afirmaram comer carne crua/mal passada, enquanto que 91,2\% faziam o consumo de verduras cruas. Assemelhando-se a esses resultados, em Maringá-PR de 150 manipuladores 96\% faziam a ingesta de verduras cruas, enquanto que 
divergindo, desses achados, em Parnaíba-PI de 251 manipuladores $62,5 \%$ consumiam carne crua ${ }^{6,18}$. O alto consumo de vegetais crus pelos manipuladores de merenda escola de Cuité-PB pode ser um achado preocupante, levando em consideração que $40 \%$ deles lavavam verduras de forma incorreta; dessa forma, a ingesta desse tipo de alimento, quando mal higienizado, pode ocasionar em contaminação por helmintos e protozoários $^{16}$. A mesma preocupação é dada àqueles que fazem o consumo de carne crua e/ou mal passada, uma vez que o consumo desse alimento contaminado com larvas cisticercoides pode propiciar o desenvolvimento da teníase ${ }^{29}$.

Quanto ao hábito de andar descalço, 14\% ( $\mathrm{n}=8)$ dos manipuladores, do município de Cuité-PB afirmaram possui-lo, sugestão de redação: No município de CuitéPB 14\% $(n=8)$ dos manipuladores afirmaram ter o hábito de andar descalço, enquanto que em Maringá-PR, de 150 manipuladores, somente $6 \%$ relataram ter costume de andar descalços ${ }^{18}$. Mesmo não sendo constatada a presença de Strongyloidesstercoralis ou Ancylostomidae, nas amostras analisadas, o hábito de andar descalço é um fator de risco para a contaminação a partir desses tipos de parasitos ${ }^{29}$.

Sobre a capacitação desses profissionais, em Cuité-PB, 42,9\% nunca participaram de nenhum tipo de treinamento relacionado à manipulação de alimentos. Enquanto que em seis municípios distintos do Estado do Rio de Janeiro, de 103 funcionários, 30,1\% nunca tiveram treinamento ${ }^{30}$.

A falta de capacitação por parte considerável dos manipuladores está em desacordo com a lei de Qualidade Alimentar do Estado da Paraíba n ${ }^{0}$ 7.587/2004 que delimita a obrigatoriedade da realização do curso básico de manipulação de alimentos, com validade de até dois anos. Ademais, obriga-se ainda que o EPF seja feito a cada seis meses, entretanto tal exigência foi seguida somente por $12,3 \%$ desses profissionais ${ }^{31}$.

\section{CONCLUSÃO}

O perfil de parasitados foi predominantemente do sexo feminino, com faixa etária de 20 a 49 anos de idade, baixa escolaridade e baixa renda. No geral, os manipuladores viviam em precárias condições sanitárias, apresentando esgotamento sanitário majoritariamente inadequado e água de abastecimento inadequada/sem procedência tanto no ambiente de trabalho quanto domiciliar.

Observou-se alta prevalência de infectados por enteroparasitos/enterocomensais (78,9\%) entre os manipuladores de merenda escolar analisados, na qual Endolimaxnana e Endolimaxnana e Entamoebacoli foram as espécies parasitárias mais encontradas nas amostras fecais positivas. Ademais, o encontro das espécies patogênicas Entamoeba histolytica/E.dispar, G. lamblia, H. nana e A. lumbricoides foi considerado um fator preocupante pelo seu potencial de patogenicidade e de transmissão interpessoal e ambiental.

Desta forma, a capacitação dos manipuladores de merenda escolar associada à realização de exames parasitológicos periódicos devem ser realizadas, a fim de permitir maior nível de consciência acerca da possível contaminação de alimentos e permitir a quebra de cadeia de transmissão de enteroparasitos. Além disso, melhorias sanitárias tornam-se necessárias, uma vez que a precariedade de saneamento básico age como facilitadora para o desenvolvimento de enteroparasitoses e outras enfermidades.

\section{REFERÊNCIAS}

1. Brasil. Ministério da Saúde. Doenças Transmitidas por Alimentos (DTA) [Internet]. Brasília; 2014. [updated 2014; cited 2018 July 13]. Available from:http://portalsaude.saude.gov.br/index.php/ o-ministerio/principal/secretarias/svs/doencastransmitidas-por-alimentos-dta

2. Cunha LF, Amichi KR. Relação entra a ocorrência de enteroparasitoses e práticas de higiene de manipuladores de alimentos: Revisão da Literatura. Rev Saúde Pesquisa. 2014;7 (1):147-57.

3. Porto LP, Cavagnolli NI, Reis DS, Spada PKWD, Rodrigues $\mathrm{AD}$. Prevalência de parasitoses em trabalhadores de restaurantes de Caxias do Sul RS. Rev Patol Trop. 2016; 45(1): 115-20.https://doi. org/10.5216/rpt.v45i1.39980. 
4. Andrade EC, Leite ICG, Rodrigues VO, Cesca MG. Parasitoses intestinais: uma revisão sobre seus aspectos sociais, epidemiológicos, clínciso e terapêuticos. Rev APS. 2010; 13(2): 231-40.

5. Lima DS, Mendonça RA, Dantas FCM, Brandão JOC, Medeiros CSQ. Parasitoses intestinais infantis no Nordeste brasileiro: uma revisão integrativa da literatura. Cadernos de Graduação - Ciências Biológicas e da Saúde Facipe. 2013;1(2): 71-80.

6. Fernandes NS, Guimarães HR, Amorim ACS, Brito VM, Borges EP, Reis MB, Trindade RA, Melo ACFL. Ocorrência de enteroparasitoses em manipuladores de alimentos de restaurantes em Parnaíba, PiauíBrasil. Rev Patol Trop. 2014;43(4): 459-69.https:// doi.org/10.5216/rpt.v43i4.33614

7. Anvisa. Agência Nacional de Vigilância Sanitária, Brasil. Resolução RDC n ${ }^{0} 216$, de 15 de setembro de 2004 [Internet]. Brasília; 2004 [updated 2004; cited 2018 August]. Avialable from: .

8. Gonçalves ALR, Belizário TL, Pimentel JB, Penatti MPA, Pedroso RS. Prevalence of intestinal parasites in preschool children in the region of Uberlândia, State of Minas Gerais, Brazil. Rev Soc Bras Med Trop. 2011;44(2): 191-93. http://dx.doi.org/10.1590/ S0037-86822011005000022

9. Fernandes S, Beorlegui M, Brito MJ, Rocha G. Protocolo de parasitoses intestinais. Acta Pediátrica Portuguesa. 2012;43(1): 35-41.

10. Santos YKA, Barbosa, VSA. Prevalência e fatores de risco para enteroparasitoses em manipuladores de alimentos da cidade de Cuité-PB. In: CONGRESSO DE INICIAÇÃO CIENTÍFICA DA UFCG, 13, 2016, Campina Grande. Anais eletrônicos. Campina Grande: Universidade Federal de Campina Grande, 2016 [Internet]. Available from: http://pesquisa.ufcg. edu.br/anais/2016/resumos/xiii-cicufcg-4281.pdf

11. Luna FB. Sequência básica na elaboração de protocolos de pesquisa. Arq Bras Cardiol. 1998;71(6): 735-40.

12. Ibeg. Instituto Brasileiro de Geografia e Estatística. Cidades - Cuité [Internet]. 2017 [updated 2017; cited 2018 Feb 19]. Available from: https://cidades. ibge.gov.br/brasil/pb/cuite/panorama

13. Castro JM, Lopes FF, Cochi ICR, Santos SV. Centrifugo-sedimentação simples: uma alternativa de substituição ao método de ritchie. In: Congresso da sociedade paulista de parasitologia, 03, 2006, Ubatuba. Anais eletrônicos. Ubatuba: UNICAMP, 2006 [Internet]. 2006 [updated 2006; cited 2018 august 10]. Available from: https://www2. ib.unicamp.br/branco/parasit/spp/congressolll/ trabalhos/96TECNO.doc

14. De Carli, GA. Parasitologia Clínica. 2. ed. São Paulo: Atheneu, 2006.

15. Agevisa. Agência Estadual de Vigilância Sanitária. Lei $\mathrm{n}^{0} 7587$, de 02 de junho de 2004 [Internet]. João Pessoa; 2004 [updated 2004; cited 2018 July 05]. Available from: http://imeq.pb.gov.br/agevisa/ documentos-pdf/legislacao/leis/lein_7587.pdf

16. Simões J, Aleixo DL. Prevalência de enteroparasitoses em manipuladores de alimentos de escolas municipais de Campo Mourão - Paraná. Sabios. 2014;9(1): 75-85.

17. Moura ACC, Avelar DM. Enteroparasitos em manipuladores de alimentos de algumas escolas públicas das cidades de luz e dores do indaiá, Minas Gerais, Brasil. Science in Health. 2013;4(3): 138-46.

18. Colli CM, Mizutani AS, Martins VA, Ferreira EC, Gomes ML. Prevalence and risk factors for intestinal parasites in food handlers, southern Brazil. Int $\mathrm{J}$ Environ Health Res. 2013;24(5): 450-458. http:// dx.doi.org/10.1080/09603123.2013.857392

19. Capuano DM, Lazzarini MPT, Giacometti Júnior E, Takayanagui OM. Enteroparasitoses em manipuladores de alimentos do município de Ribeirão Preto - SP, Brasil, 2000. Rev Bras Epidemiol. 2008;11(4): 687-95.

20. Takizawa MGMH, Falavigna DLM, Gomes ML. Enteroparasitosis and their ethnographic relationship to food handlers in a touris and economic center in Paraná, southern Brazil. Rev Inst Med Trop S. Paulo. 2009; 51(1): 31-35. 
21. Reis RM, Carneiro LC. Indicador higiênico-parasitário em manipuladores de alimentos em Morrinhos, GO. Estudos de Biologia. 2007;29(68): 313-17.

22. Silva EJ, Silva RMG, Silva LP. Investigação de parasitos e/ou comensais intestinais em manipuladores dealimentos de escolas públicas. Biosci J. 2009;25(4): 160-63.

23. Lima Júnior WA, Kaiser J, Catisti R. High occurence of giardiasis in children living on a "Landless farm workers" settlement in Arara, São Paulo, Brasil. Rev Inst Med Trop S Paulo. 2013;55(3): 185-88.

24. Magalhães VM, Carvalho AG, Freitas, FIS. Inquérito parasitológico em manipuladores de alimentos em João Pessoas, PB, Brasil. Rev Patol Trop. 2010;39(4): 335-42. https://doi.org/10.5216/rpt.v39i4.13069

25. Sande S, Basak S, Sande V, Tawade V. Screening of food handlers for intestinal parasites and enteropathogenic bacteria in a tertiary care hospital. International Journal of Health Sciences \& Research. 2014; 4(5): 88-94.

26. Franco CR, Ueno M. Comércio ambulante de alimentos: condições higiênico-sanitárias nos pontos de venda de Taubaté-SP. Unopar Científica Ciências Biológicas e da Saúde. 2010;12(4): 9-13.

27. Nunes XP, Almeida JRGS, Nunes XP. Anemia ferropriva, enteroparasitoses e esgotamento sanitário. Rev bras pesq saúde. 2014;16(1): 118-24.

28. Ely LS, Engroff P, Lopes GT, Werlang M, Gomes I, Carli GA. Prevalência de enteroparasitos em idosos. Rev Bras Geriatr Gerontol. 2011;14(4): 637-46. http:// dx.doi.org/10.1590/\$1809-98232011000400004

29. Neves DP. Parasitologia Humana. 13. ed. São Paulo: Atheneu; 2016.

30. Mello AG, Gama MP, Marin VA, Colares LGT. Conhecimento dos manipuladores de alimentos sobre boas práticas nos restaurantes públicos populares do Estado do Rio de Janeiro. Braz J Food Technol. 2010;13(1): 60-68.

31. Brasil. Agência Estadual de Vigilância Sanitária. Lei $\mathrm{n}^{\mathrm{o}} 7587$, de 02 de junho de 2004 [Internet]. 2004 [updated 2004; cited 2018 July 05]. Available from: http://imeq.pb.gov.br/agevisa/documentos-pdf/ legislacao/leis/lein_7587.pdf

32. Kheirandish F., Tarahi MJ, Ezatpour B. Prevalence of intestinal parasites among food handlers in western Iran. Rev. Inst. Med. Trop. São Paulo. 2014; 56(2): 111-4.

Recebido em: 04/01/2019

Aceito em: 20/08/2019 